\title{
Diagnostic performance of the Luminex XTAG gastrointestinal pathogens panel to detect rotavirus in Ghanaian children with and without diarrhoea
}

Amelie Leva ${ }^{1}$, Daniel Eibach², Ralf Krumkamp ${ }^{2,3}$, Julia Käsmaier², Dennis Rubbenstroth', Yaw Adu-Sarkodie ${ }^{4}$, Jürgen May ${ }^{2,3}$, Egbert Tannich ${ }^{2,3}$ and Marcus Panning ${ }^{1 *}$

\begin{abstract}
Background: Rotavirus is one of the leading causes of childhood diarrhoea worldwide. The highest disease burden is seen in resource-constrained settings of sub-Saharan Africa. Recently, commercial multiplex PCR panels proved their accuracy to diagnose infectious gastroenteritis in Europe and the USA. However, data on their performance using samples from tropical regions in general and to detect rotavirus in particular remains scant. We aimed to analyse the diagnostic performance of the Luminex xTAG gastrointestinal pathogens panel, a multiplex PCR, to detect rotavirus in stool samples from Ghanaian children.

Methods: A total of 682 stool samples were collected in the Ashanti region of Ghana between 2007 and 2008. Of these, 341 were from cases (children with diarrhoea), and another 341 from controls (children without diarrhoea). All samples were analysed using the Luminex XTAG assay and compared to a rotavirus quantitative reverse-transcription PCR (reference assay). Rotavirus reference assay positive samples were $P$ and $G$ genotyped by sequencing the rotavirus VP4 and VP7 genes.

Results: Overall agreement between the Luminex XTAG and the reference assay was excellent (kappa 0.93). The sensitivity and specificity was $88.2 \%$ (95 \% confidence interval [Cl] 78.2-94.1) and $100 \%$ (95 \% Cl 99.2-100), respectively. Of 76 rotavirus reference assay positive samples, 64 were successfully genotyped and the Luminex xTAG assay was able to detect all rotavirus genotypes present in the study.

Conclusion: The Luminex XTAG assay proved a sensitive and highly specific tool to detect rotavirus and may aid clinicians and public health authorities in the diagnosis and surveillance of rotavirus.
\end{abstract}

Keywords: Rotavirus, Luminex xTAG gastrotinestinal pathogens panel, Stool samples, Performance, Sub-saharan Africa

\section{Background}

Rotavirus gastroenteritis is a major public health threat responsible for a high disease burden in low-income countries [1]. Laboratory diagnosis of rotavirus gastroenteritis is traditionally accomplished using enzymeimmunoassays (EIA) and current EIA kits demonstrated a good overall performance [2]. However, PCR methods

\footnotetext{
* Correspondence: marcus.panning@uniklinik-freiburg.de

${ }^{1}$ Institute for Virology, Center for Microbiology and Hygiene, University Medical Center - Freiburg University, Hermann-Herder-Str. 1179104, Freiburg, Germany

Full list of author information is available at the end of the article
}

have proven their advantages over conventional methods mainly due to a higher analytical sensitivity $[3,4]$. Commercial multiplex PCR panels became available recently allowing the simultaneous detection of several targets including rotavirus. These panels were already successfully evaluated in Europe, the USA, and more recently in Vietnam [5-7]. We applied the Luminex xTAG gastrointestinal pathogens panel (GPP) in a rural African setting and could demonstrate a high rate of positive stool samples [8]. The assay was applied to diagnose gastrointestinal pathogens in children with and without diarrhoea to evaluate the usefulness of the GPP. Overall the 
three most common pathogens were enterotoxigenic Escherichia coli, Giardia lamblia, and Shigella spp.. In particular, we could show an association with diarrhoea for rotavirus. However, there is only scant evidence on the diagnostic performance of commercial multiplex PCR panels using samples from tropical settings in general and to detect rotavirus in particular.

We aimed to evaluate the performance of the Luminex xTAG GPP multiplex PCR in comparison to a reference quantitative reverse-transcription PCR (qRT-PCR) to detect rotavirus in stool samples from Ghanaian children.

\section{Results}

Among cases, 49/341 (14.4 \%) tested positive using qRTPCR, and 44/341 (12.9\%) tested positive using the GPP. In controls, 27/341 (7.9\%) were qRT-PCR positive, and 23/341 (6.7 \%) were positive using the GPP assay. Comparable qRT-PCR Ct-values between cases (median Ct-value: 21.5, interquartile range [IQR]: 19.8; 25.1) and controls (median Ct-value: 22.0, IQR: 19.2; 27.0) were observed $(p=0.66)$. Rotavirus was detected more frequently in cases $(n=49,14.4 \%)$ compared to controls $(n=27$, $7.9 \%)$ using qRT-PCR $(p=0.010)$, and GPP $(n=44$, $12.9 \%$ versus $n=23,6.7 \% ; p=0.009$ ), respectively.

Overall agreement between qRT-PCR and GPP was excellent (kappa 0,93). The test performance of the GPP in comparison to qRT-PCR is summarized in Table 1. In cases, the sensitivity of GPP was slightly higher (Table 1). The nine GPP false-negative results had a median qRTPCR Ct-value of 30 (IQR: 28.8; 32.5) indicating low rotavirus loads. Of note, all nine samples tested rotavirus positive using the FTD viral gastroenteritis assay, suggesting that these samples were indeed GPP falsenegatives. Five of these nine samples were from cases, and four from controls.

There was a significant difference in qRT-PCR Ctvalues between 44 GPP-positive (median Ct-value: 21, IQR: 19.8; 23.3) and five GPP-negative cases (median Ct-value: 29, IQR: 27.5; 29.8, $p=0.0001$ ) (Fig. 1). Of the 76 qRT-PCR positive samples 64 (84\%) were rotavirus $\mathrm{G}$ and P genotyped. The GPP assay was able to detect all rotavirus genotypes present in the study (Table 2). Of note, we failed to genotype the nine GPP false-negative samples.

Comparable MFI-values using the GPP assay between cases (median MFI: 3875; IQR: 2791; 4795) and controls (median MFI: 3314, IQR: 2173; 4485) were observed $(p=0.33)$. Next, we analyzed GPP MFIvalues in four different groups with qRT-PCR Ctvalues <20 (median MFI: 4475, IQR: 3766; 4960), 2025 (median MFI: 3505, IQR: 2583; 4414), 25-30 (median MFI: 1694, IQR: 145; 2882), and >30 (median MFI: 46, IQR: 44; 63), respectively (Fig. 2). There was a statistically significant difference between the groups with Ct-values ranging from 20-25, 25-30, and >30, respectively, using one-way ANOVA analysis. Only a weak negative correlation of $-0.47(95 \% \mathrm{CI}-6.64$ to -0.26) was seen between qRT-PCR values and GPP MFI-values.

Finally, the number of co-detections with other pathogens as determined using the GPP did not differ significantly between cases (median 3, IQR: 2; 3) and controls (median 2, IQR: 1; 4), and between GPP-positive (median 2, IQR: 1; 3) and GPP-negative (median 2, IQR: 2; 4) samples. Overall the rate of co-detections was statistical significantly different between rotavirus GPPpositive [67/67 (100 \%)] and GPP-negative [575/615 $(93.5 \%)]$ samples (Fishers exact test, $p=0.025$ ). The distribution of pathogens among rotavirus GPP-positive and rotavirus GPP-negative samples is shown in Table 3. Of note, significantly more enterotoxigenic Escherichia coli (59 \% versus $43 \%, p=0,0187)$, Escherichia coli O157 (15\% versus $3 \%, p=0,0029)$, and Shigella spp. (40\% versus $22 \%, p=0,0052)$ were detected among rotavirus GPP-negative samples compared to GPPpositive samples (Table 3 ).

\section{Discussion}

We could show an excellent agreement between the GPP and qRT-PCR to detect rotavirus in stool samples from Ghanaian children. The high specificity of the GPP is reassuring. The sensitivity of the GPP to detect rotavirus was slightly lower than reported previously $[5,9]$. However, numbers of rotavirus positive samples were rather low in both studies [5, 9]. Another study from Vietnam used a comprehensive panel of samples and demonstrated a sensitivity of $92.2 \%$ and specificity of $98.9 \%$, respectively, for rotavirus [7]. These data from an Asian tropical country are in line with our findings although different rotavirus qRT-PCR assays were used as a comparator. On the other hand, rare rotavirus genotypes or genetic variants which are prevalent in West Africa might undergo GPP detection, but warrants further studies [10]. As a limitation we could not sequence

Table 1 Performance of the Luminex GPP in comparison to the rotavirus reference qRT-PCR assay among all study subjects (top panel) and cases only (bottom panel)

\begin{tabular}{llllllll}
\hline & TP, $n$ & FP, $n$ & FN, $n$ & TN, $n$ & Sensitivity \% (95 \% Cl) & Specificity \% (95 \% Cl) & kappa \\
\hline All study subjects $(n=682)$ & 67 & 0 & 9 & 606 & $88.2(78.2-94.1)$ & $100(99.2-100)$ & 0.93 \\
Cases $(n=341)$ & 44 & 0 & 5 & 292 & $89.7(76.9-96.2)$ & $100(98.4-100)$ & 0.94 \\
\hline
\end{tabular}

$T P$ true positive; $F P$ false positive; $F N$ false negative; $T N$ true negative 


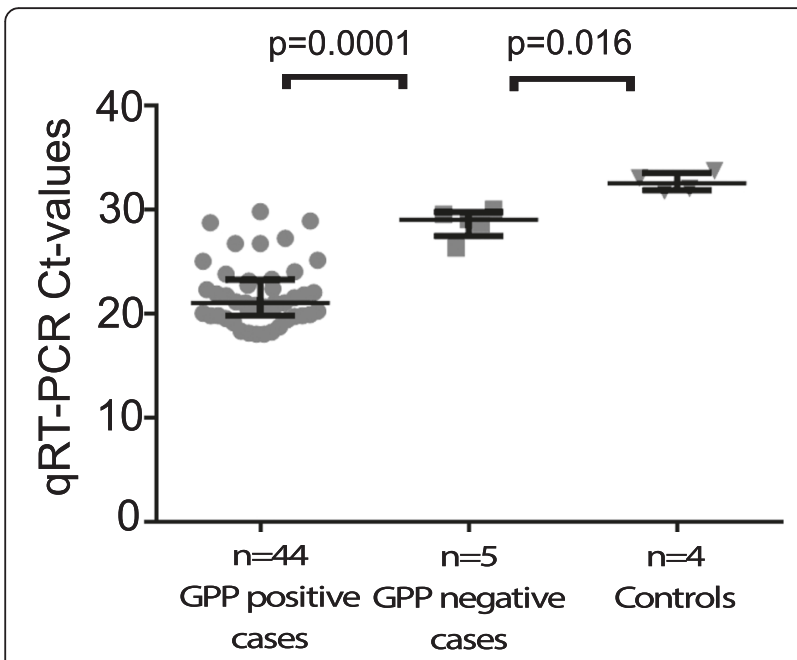

Fig. 1 Distribution of qRT-PCR cyle threshold values. Shown are qRT-PCR cycle threshold values in GPP-positive and GPP-negative samples among cases and controls

the qRT-PCR positive/GPP negative samples mainly due to low virus concentrations. Little left over material prevented further sequencing efforts.

Intriguingly, we detected less rotavirus shedding among controls using GPP and qRT-PCR compared to a recent study from Malawi [11]. Differences in herd immunity are possible reasons for this finding. However, rotavirus shedding using RT-PCR has been observed in different studies but the relevance remains unclear [12]. Subclinical infections or prolonged shedding after acute infections might play a role and deserve further studies. In light of the high sensitivity of the qRT-PCR and consistency with GPP results it is less likely that sensitivity issues played a role. Interestingly, the median qRTPCR Ct-value of GPP-positive cases in our study is close to the Ct-value of 19.5 in diarrheal cases of another study supporting the notion that low Ct-values are associated with clinically relevant disease [11]. Of note, the results of the GPP are of qualitative nature only and quantitative results might provide a better resolution of

Table 2 Shown are results for the Luminex GPP assay according to rotavirus genotype and median Ct-value of qRT-PCR

\begin{tabular}{llll}
\hline $\begin{array}{l}\text { Rotavirus } \\
\text { genotype }\end{array}$ & $\begin{array}{l}\text { No. of } \\
\text { strains }\end{array}$ & $\begin{array}{l}\text { Median qRT-PCR Ct- } \\
\text { value, (IQR) }\end{array}$ & $\begin{array}{l}\text { Luminex GPP } \\
\text { positive, } n=(\%)\end{array}$ \\
\hline G1[P8] & 25 & $20.3(19.3-22.8)$ & $25(100)$ \\
G2[P4] & 17 & $21(19.3-22.6)$ & $17(100)$ \\
G2[P6] & 12 & $20.4(18.8-21.9)$ & $12(100)$ \\
G2[P-UD $\left.{ }^{a}\right]$ & 7 & $25.1(21.7-27.2)$ & $7(100)$ \\
G3[P6] & 3 & $22.1(20-23.1)$ & $3(100)$ \\
Total & 64 & $21(19.6-23.3)$ & $64(100)$ \\
\hline
\end{tabular}

Abbreviations: IQR interquartile range; $C t$ cycle threshold, GPP gastrointestinal pathogens

${ }^{a}$ UD, undetermined

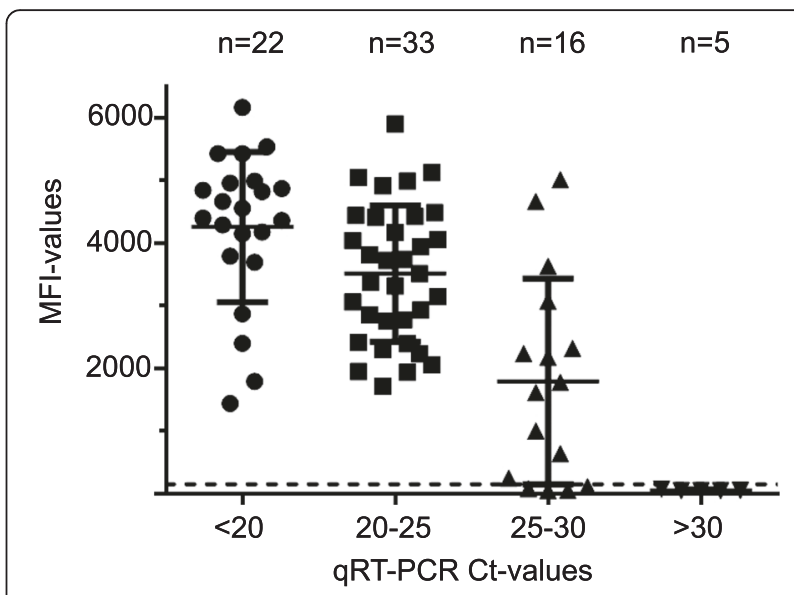

Fig. 2 MFl-values according to qRT-PCR cycle threshold values. Shown are MFI-values using the GPP assay in four different groups with qRT-PCR Ct-values <20, 20-24, 25-29, and $>30$. The horizontal dotted line indicates the GPP threshold of $150 \mathrm{MFI}$ for positivity

PCR results and disease [13]. As a surrogate marker for rotavirus RNA concentrations we could show that MFI values of the GPP might provide useful semiquantitative information. However, further studies are needed to establish reliable quantitative data using the GPP. Ultimately, studies are needed to appreciate the value of multiplex PCR in order to improve patient care. As a limitation of our study we did not address the diagnostic performance of all pathogens included in the GPP panel. Previous studies have shown a good overall performance but further studies using samples from Africa are needed.

\section{Conclusion}

We evaluated the Luminex GPP assay to detect rotavirus using stool samples from Ghanaian children and could demonstrate that the Luminex GPP is a sensitive and highly specific tool for this purpose. The GPP is able to detect a broad range of rotavirus genotypes prevalent in Ghana and our results suggest that the assay can provide semi-quantitative data which requires further investigation. Thus, pending its implementation in resourceconstrained African countries the GPP assay might provide a valuable tool in the detection and surveillance of rotavirus.

\section{Methods}

Samples used in the current study were a subset of samples collected in the framework of a case-control study on causes of diarrhoea in children. The study was conducted at the children's Outpatients Department (OPD) of the Agogo Presbyterian Hospital, a district hospital in the Ashanti region of Ghana [14]. For the current study, stool samples from children below 6 years of age visiting the hospital's OPD between June 2007 and October 2008 
Table 3 Number and percentage of co-detections among rotavirus GPP-positive and rotavirus GPP-negative samples

\begin{tabular}{llll}
\hline & Rotavirus GPP-positive, $n=67$ & Rotavirus GPP-negative, $n=615$ \\
Pathogen & $n=,(\%)$ & $n=,(\%)$ & $p$-value* \\
\hline Adenovirus 40/41 & $4(6)$ & $27(4)$ & 0,5338 \\
Norovirus GI/GIl & $3(4)$ & $63(10)$ & 0,1884 \\
Campylobacter spp. & $19(28)$ & $212(34)$ & 0344 \\
Clostridium difficile toxin A/B & $1(1)$ & $3(0,5)$ & 0,3394 \\
Cryptosporidium spp. & $1(1)$ & $44(7)$ & 0,1137 \\
Entamoaba histolytica & $0(0)$ & $3(0,5)$ & 1 \\
Escherichia coli LT/ST (ETEC) & $29(43)$ & $362(59)$ & 0,0187 \\
Escherichia coli O157 & $2(3)$ & $94(15)$ & $20(3)$ \\
Escherichia coli STEC (stx1/2) & $1(1)$ & $325(53)$ & 0,0029 \\
Giardia lamblia & $28(42)$ & $84(14)$ & 0,7115 \\
Salmonella spp. & $6(9)$ & $246(40)$ & 0,0947 \\
Shigella spp. & $15(22)$ & $0(0)$ & 0,3445 \\
Yersinia enterocolitica & $0(0)$ & $0(0)$ & 0,0052 \\
Vibrio cholerae & $0(0)$ & & 1 \\
\hline
\end{tabular}

*Fisher's exact test

were selected. Children with diarrhoea, defined as at least three loose stools within the last $24 \mathrm{~h}$, served as cases. During the same period, children attending the OPD without gastrointestinal symptoms were recruited as controls. A total of 682 samples were selected for this study. Of these, 341 samples were from cases and another 341 were from controls.

Immediately after stool collection, the samples were frozen at $-20{ }^{\circ} \mathrm{C}$ and shipped on dry ice to Germany for further analyses as described elsewhere [14]. Following nucleic acid extraction from $200 \mathrm{mg}$ stool samples were analyzed using the GPP (Luminex Corporation, Austin, TX) according to the manufacturer's instructions. The results for rotavirus are reported in median fluorescence intensity (MFI) values from each sample. A published rotavirus qRT-PCR was used as the reference assay [15]. Briefly, a qRT-PCR targeting the NSP3 gene was used to detect rotavirus nucleic acids. A $25-\mu \mathrm{l}$ reaction contained $5 \mu \mathrm{l}$ of prepared nucleic acids (the same as used for the GPP), $2 \times$ AgPath-ID One-Step RT-PCR buffer (Thermo Fisher, Darmstadt, Germany), 0.4 $\mu \mathrm{M}$ primer JVKF (CAGTGGTTGATGCTCAAGATGGA; TIB-Molbiol, Berlin, Germany), $0.4 \mu \mathrm{M}$ primer JVKR (TCATTGTAATCATATTGAATACCCA, TIB-Molbiol), $0.2 \mu \mathrm{M}$ probe JVKP (ACAACTGCAGCTTCAAAAGAAGWGT). Probe JVKP was labeled with 5' FAM and a $3^{\prime}$ nonfluorescent quencher (TIB-Molbiol). The cycling conditions in an ABI Prism 7500 machine (Thermo Fisher) were as follows: $50{ }^{\circ} \mathrm{C}$ for $15 \mathrm{~min}, 95{ }^{\circ} \mathrm{C}$ for $10 \mathrm{~min}$, and 45 cycles of $95{ }^{\circ} \mathrm{C}$ for $10 \mathrm{~s}$ and $60{ }^{\circ} \mathrm{C}$ for $35 \mathrm{~s}$. The data were analyzed with the Sequence detector software V 2.0.6 (Thermo Fisher). Positive and no-template controls were included in each PCR run.
The analytical sensitivity of the qRT-PCR was 12.5 in vitro-transcribed RNA copies per reaction [95\% confidence interval (CI) 9.6 to 20.9 RNA copies per reaction] as determined using probit analysis. The rotavirus strains were $G$ and $P$ genotyped by sequencing according to Iturizza-Gomara et al. [16]. The Fast Track Diagnostics (FTD) viral gastroenteritis kit (Junglingster, Luxemburg) was used to analyze discrepant results between GPP and qRT-PCR. A sample was considered GPP false-positive if both the qRT-PCR and the viral gastroenteritis kit yielded a negative rotavirus result.

All statistical analyses were done using the GraphPad Prism software package (GraphPad, San Diego, CA, USA). Median cycle threshold (Ct)-values and MFI-values between two groups was compared using Wilcoxon rank sum test. Differences in the detection frequency of rotavirus using GPP and qRT-PCR in cases and controls were assessed with Fisher's exact test. A $p$-value of $<0.05$ was considered statistically significant. Correlation between qRT-PCR Ct-values and GPP MFI-values was assessed using the Pearson correlation coefficient. The Committee on Human Research, Publications and Ethics, School of Medical Science, Kwame Nkrumah University of Science and Technology, Kumasi, Ghana, approved the study design and the informed consent procedure.

\section{Abbreviations}

$\mathrm{Cl}$, confidence interval; Ct-value, cycle threshold value; EIA, enzyme-linked immunosorbent assay; GPP, gastrointestinal pathogens panel; IQR, interquartile range; MFI, mean fluorescence intensity; OPD, Outpatients Department; qRT$P C R$, quantitative reverse-transcription polymerase chain reaction. 


\section{Acknowledgements}

We are grateful to Björn Eberle for excellent technical assistance.

\section{Funding}

This work was supported by UBS Optimus Foundation.

\section{Authors' contribution}

$J M, E T$, and MP designed the study. JK, YAS, ET conducted and supervised field work. AL and JK carried out laboratory analysis. DR performed phylogenetic analysis. AL, RK, DE, JM, ET, and MP performed the data analysis and drafted the manuscript. All authors participated in writing and revising the manuscript. All authors have read and approved the final manuscript.

\section{Competing interests}

The authors declare that they have no competing interests.

\section{Ethical approval}

The Committee on Human Research, Publications and Ethics, School of Medical Science, Kwame Nkrumah University of Science and Technology, Kumasi, Ghana, approved the study design and the informed consent procedure.

\section{Author details}

'Institute for Virology, Center for Microbiology and Hygiene, University Medical Center - Freiburg University, Hermann-Herder-Str. 1179104, Freiburg, Germany. ${ }^{2}$ Bernhard Nocht Institute for Tropical Medicine, Bernhard-Nocht-Str. 74, 20359 Hamburg, Germany. ${ }^{3}$ German Center for Infection Research (DZIF), Hamburg-Borstel-Lübeck, Hamburg, Germany. ${ }^{4}$ Kwame Nkrumah University of Science and Technology (KNUST), Kumasi, Ghana.

Received: 13 May 2016 Accepted: 24 July 2016

Published online: 29 July 2016

\section{References}

1. Tate JE, Burton AH, Boschi-Pinto C, Steele AD, Duque J, Parashar UD, Network WH-cGRS. estimate of worldwide rotavirus-associated mortality in children younger than 5 years before the introduction of universal rotavirus vaccination programmes: a systematic review and meta-analysis. Lancet Infect Dis. 2008;2012(12):136-41.

2. Gautam R, Lyde F, Esona MD, Quaye O, Bowen MD. Comparison of Premier Rotaclone(R), ProSpecT, and RIDASCREEN(R) rotavirus enzyme immunoassay kits for detection of rotavirus antigen in stool specimens. J Clin Virol. 2013; 58:292-4.

3. Amar CF, East CL, Gray J, Iturriza-Gomara M, Maclure EA, McLauchlin J. Detection by PCR of eight groups of enteric pathogens in 4,627 faeca samples: re-examination of the English case-control Infectious Intestinal Disease Study (1993-1996). Eur J Clin Microbiol Infect Dis. 2007;26:311-23.

4. Esona MD, Gautam R. Rotavirus. Clin Lab Med. 2015;35:363-91.

5. Navidad JF, Griswold DJ, Gradus MS, Bhattacharyya S. Evaluation of Luminex xTAG gastrointestinal pathogen analyte-specific reagents for highthroughput, simultaneous detection of bacteria, viruses, and parasites of clinical and public health importance. J Clin Microbiol. 2013:51:3018-24.

6. Mengelle C, Mansuy JM, Prere MF, Grouteau E, Claudet I, Kamar N, Huynh A, Plat G, Benard M, Marty N, et al. Simultaneous detection of gastrointestinal pathogens with a multiplex Luminex-based molecular assay in stool samples from diarrhoeic patients. Clin Microbiol Infect. 2013;19:E458-465.

7. Duong VT, Phat W, Tuyen HT, Dung TT, Trung PD, Minh PV, TP TI, Campbell II, Le Phuc $\mathrm{H}, \mathrm{Ha} T \mathrm{~T}$, et al. Evaluation of Luminex XTAG gastrointestinal pathogen panel assay for detection of multiple diarrheal pathogens in fecal samples in Vietnam. J Clin Microbiol. 2016;54:1094-100

8. Eibach D, Krumkamp R, Hahn A, Sarpong N, Adu-Sarkodie Y, Leva A, Kasmaier J, Panning M, May J, Tannich E. Application of a multiplex PCR assay for the detection of gastrointestinal pathogens in a rural African setting. BMC Infect Dis. 2016;16:150

9. Claas EC, Burnham CA, Mazzulli T, Templeton K, Topin F. Performance of the xTAG(R) gastrointestinal pathogen panel, a multiplex molecular assay for simultaneous detection of bacterial, viral, and parasitic causes of infectious gastroenteritis. J Microbiol Biotechnol. 2013;23:1041-5.
10. Mwenda JM, Tate JE, Parashar UD, Mihigo R, Agocs M, Serhan F, Nshimirimana D. African rotavirus surveillance network: a brief overview. Pediatr Infect Dis J. 2014;33 Suppl 1:S6-8.

11. Bennett A, Bar-Zeev N, Jere KC, Tate JE, Parashar UD, Nakagomi O, Heyderman RS, French N, Iturriza-Gomara M, Cunliffe NA. Determination of a viral load threshold to distinguish symptomatic versus asymptomatic rotavirus infection in a high-disease-burden African population. J Clin Microbiol. 2015;53:1951-4

12. Corcoran MS, van Well GT, van Loo $\mathbb{H}$. Diagnosis of viral gastroenteritis in children: interpretation of real-time PCR results and relation to clinical symptoms. Eur J Clin Microbiol Infect Dis. 2014;33:1663-73.

13. Liu J, Kabir F, Manneh J, Lertsethtakarn P, Begum S, Gratz J, Becker SM, Operario DJ, Taniuchi M, Janaki L, et al. Development and assessment of molecular diagnostic tests for 15 enteropathogens causing childhood diarrhoea: a multicentre study. Lancet Infect Dis. 2014;14:716-24.

14. Krumkamp R, Sarpong N, Schwarz NG, Adlkofer J, Loag W, Eibach D, Hagen RM, Adu-Sarkodie Y, Tannich E, May J. Gastrointestinal infections and diarrheal disease in Ghanaian infants and children: an outpatient casecontrol study. PLoS Negl Trop Dis. 2015;9:e0003568.

15. Jothikumar N, Kang G, Hill VR. Broadly reactive TaqMan assay for real-time RT-PCR detection of rotavirus in clinical and environmental samples. J Virol Methods. 2009:155:126-31.

16. Iturriza-Gomara M, Kang G, Gray J. Rotavirus genotyping: keeping up with an evolving population of human rotaviruses. J Clin Virol. 2004;31:259-65.

\section{Submit your next manuscript to BioMed Central and we will help you at every step:}

- We accept pre-submission inquiries

- Our selector tool helps you to find the most relevant journal

- We provide round the clock customer support

- Convenient online submission

- Thorough peer review

- Inclusion in PubMed and all major indexing services

- Maximum visibility for your research

Submit your manuscript at www.biomedcentral.com/submit
BioMed Central 